Article

\title{
Effective RNA Regulation by Combination of Multiple Programmable RNA-Binding Proteins
}

\author{
Misaki Sugimoto, Akiyo Suda, Shiroh Futaki $₫$ and Miki Imanishi * \\ Institute for Chemical Research, Kyoto University, Uji, Kyoto 611-0011, Japan; \\ sugimoto.misaki.25c@st.kyoto-u.ac.jp (M.S.); suda.akiyo.3v@kyoto-u.ac.jp (A.S.); futaki@scl.kyoto-u.ac.jp (S.F.) \\ * Correspondence: imiki@scl.kyoto-u.ac.jp; Tel.: +81-774-38-3212
}

Received: 7 September 2020; Accepted: 25 September 2020; Published: 28 September 2020

Featured Application: RNA targeting.

\begin{abstract}
RNAs play important roles in gene expression through translation and RNA splicing. Regulation of specific RNAs is useful to understand and manipulate specific transcripts. Pumilio and fem-3 mRNA-binding factor (PUF) proteins, programmable RNA-binding proteins, are promising tools for regulating specific RNAs by fusing them with various functional domains. The key question is: How can PUF-based molecular tools efficiently regulate RNA functions? Here, we show that the combination of multiple PUF proteins, compared to using a single PUF protein, targeting independent RNA sequences at the $3^{\prime}$ untranslated region (UTR) of a target transcript caused cooperative effects to regulate the function of the target RNA by luciferase reporter assays. It is worth noting that a higher efficacy was achieved with smaller amounts of each PUF expression vector introduced into the cells compared to using a single PUF protein. This strategy not only efficiently regulates target RNA functions but would also be effective in reducing off-target effects due to the low doses of each expression vector.
\end{abstract}

Keywords: RNA binding protein; PUF; RNA regulation

\section{Introduction}

Manipulation of specific RNAs is important for elucidating the roles of specific RNAs and for therapeutic purposes as represented by RNA interference and antisense strategies targeting specific mRNAs. Other than such complementary oligonucleotides, sequence-specific RNA-binding proteins, Pumilio and fem-3 mRNA-binding factor (PUF) proteins and pentatricopeptide repeat (PPR) proteins, and clustered regularly interspaced short palindromic repeats (CRISPR)-based systems, including dCas13 and dCas9-PAMer complexed with guide RNA, have been used for the customized sequence-specific regulation of mRNAs [1-6]. By combining them with various functional domains, such as translational regulation factors [7,8], splicing factors [9-11], RNA editing enzymes [12], and RNA (de)methylation enzymes [13-18], targeted control of RNAs can be achieved. The RNA-binding domains of PUF proteins consist of eight highly homologous structural repeats (repeats 1 to 8), flanked by $\mathrm{N}$ - and C-terminal repeats (repeats $\mathrm{N}$ and C) [19] (Figure 1a). Each repeat recognizes an RNA base through direct interaction between RNA bases and side chains of amino acids at the 12th and 16th positions [20] (Figure 1b). By changing the amino acid pairs of a PUF repeat, artificial RNA-binding domains corresponding to various 8-nucleotide (nt) RNA sequences can be designed [9,21,22]. As the length of RNA recognized by PUFs (8-nt) is not enough to specify the target RNAs within a huge transcriptome, efforts to increase the length of the RNA-binding sequences have been applied by increasing the number of PUF repeats [21,23-26]. Previous reports also showed the assembly of two PUF proteins in adjacent regions on the RNA to image specific 
transcripts [27-30]. By contrast, Cao et al. reported that increasing the number of the PUF-binding sequences in reporter mRNAs results in the effective regulation of reporter genes compared to reporter mRNAs having a single PUF-binding sequence [7]. This was also supported through the regulation of endogenous mRNA by PUFs fused to the RNA decay factor, tristetraprolin (TTP). Abil et al. designed artificial PUF proteins, targeting the $3^{\prime}$ untranslated region (UTR) of vascular endothelial growth factor A (VEGFA) mRNA. TTP-PUF fusion proteins effectively repressed VEGFA production when the particular 8-nt PUF-binding sequence existed at multiple places within the $3^{\prime}$ UTR of the target mRNA [31]. However, it is unusual that many repeated sequences are at the control region of a single mRNA. Instead of increasing the number of target RNA sequences, we intended to use four different PUF proteins, corresponding target RNA sequences that exist at the control region of a reporter RNA (Figure 1c). In this study, we expressed PUF RNA-binding proteins fused with TTP in mammalian cells and enabled the efficient repression of the reporter protein expression by using multiple TTP-PUF proteins.

(a)

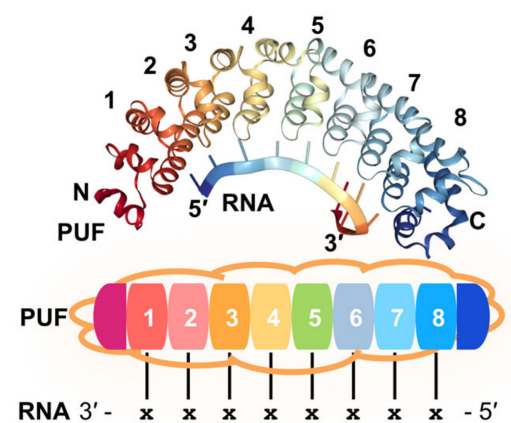

(b)

\begin{tabular}{cc}
\hline$A_{12} A_{16}$ & Base \\
\hline$(S / C) Q$ & $A$ \\
$N Q$ & $U$ \\
$S E$ & $G$ \\
SR & $C$ \\
\hline
\end{tabular}

(c)

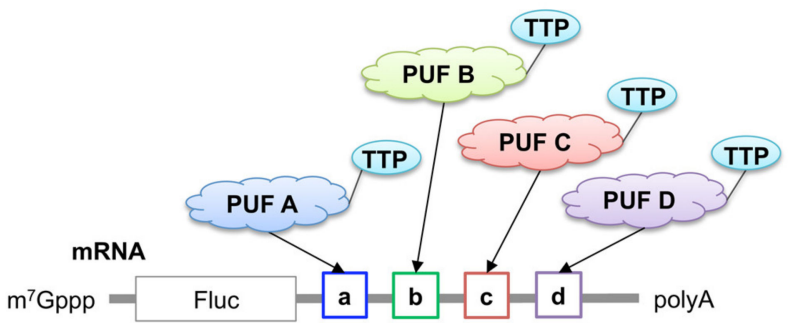

(d)

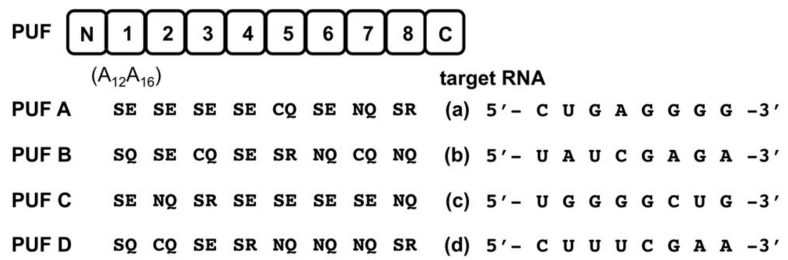

Figure 1. Programmable RNA binding protein, PUF, and regulation of RNA functions. (a) Structure of a PUF protein bound to RNA [PDB ID: 1M8Y] and schematic representation of RNA recognition by a PUF protein. (b) 12th and 16th amino acids $\left(\mathrm{A}_{12}\right.$ and $\left.\mathrm{A}_{16}\right)$ in each PUF repeat interact with an RNA base. (c) Schematic representation of mRNA regulation by multiple artificial PUF proteins fused with TTP. (d) Design of PUF proteins, PUF (A)-(D), and their recognition RNA sequences, (a)-(d), used in this research.

\section{Materials and Methods}

\subsection{Plasmid Construction}

PUF expression vectors for E. coli expression were constructed by a Golden Gate Assembly using the PUF Assembly Kit (Addgene \#1000000051) and pET28-GG-PUF as the receiving vector [31]. The amino acid sequences at the 12th and 16th positions of each repeat are shown in Figure 1d. 
TTP-PUF expression vectors for mammalian cells were constructed by Golden Gate Assembly using the PUF Assembly Kit and pCMV-TTP(C147R)-GG-PUF as the receiving vector.

Luciferase reporter vectors were constructed, as described in the text that follows. The DNA oligonucleotides containing the corresponding sequences for $4 \times \operatorname{RNA}(\mathrm{a})-(\mathrm{d})$, or $1 \times(\mathrm{a}-\mathrm{b}-\mathrm{c}-\mathrm{d})$ and their complementary DNA oligonucleotides (Eurofins genomics) were phosphorylated by $\mathrm{T} 4$ polynucleotide kinase (NEB) and annealed; they were inserted into the $3^{\prime}$ UTR of the pmirGLO vector (Promega). The sequences of $4 \times$ RNA (a)-(d), or $1 \times(a-b-c-d)$ are shown in Supplementary Figure S1.

\subsection{Protein Expression and Purification}

PUF proteins were expressed in E. coli BL21 (DE3) cells (Nippon gene) and purified as described before [23]. Briefly, protein expression was induced by adding $0.1 \mathrm{mM}$ isopropyl $\beta$-D-1-thiogalactopyranoside (IPTG), and E. coli cells were incubated at $18^{\circ} \mathrm{C}$ overnight. PUF proteins were purified from the E. coli-soluble fraction using Ni-NTA HisTrap FF column (GE Healthcare) chromatography followed by ultrafiltration by an Amicon Ultra (10 kDa NMWL) (Merck Millipore).

\subsection{Electrophoretic Mobility Shift Assay (EMSA)}

RNA oligonucleotides, labeled with 6-FAM at the 5'-end, were obtained from FASMAC. Labeled-RNA $(10 \mathrm{nM})$ and three-fold serially diluted concentrations of purified PUF proteins (0-300 nM) were mixed in the reaction buffer containing $10 \mathrm{mM}$ HEPES, $50 \mathrm{mM} \mathrm{KCl}, 0.5 \mathrm{mM}$ EDTA, $2 \mathrm{mM}$ DTT, 0.01\% Tween20, $0.1 \mathrm{mg} / \mathrm{mL}$ BSA, $0.02 \mathrm{U} / \mu \mathrm{L}$ RNasin Plus RNase Inhibitor (Promega), and 2.5\% Ficoll. The mixture was incubated at $25^{\circ} \mathrm{C}$ for $30 \mathrm{~min}$. Free RNA and bound RNA were separated via gel electrophoresis using $8 \%$ nondenaturing polyacrylamide gels in $0.5 \times \mathrm{TBE}$ at $4{ }^{\circ} \mathrm{C}$. After electrophoresis, fluorescently labeled RNAs were detected using the Typhoon scanner RGB system (GE Healthcare). The peak intensity for bound and free labeled-RNA was measured using the ImageQuant TL software (GE Healthcare). The fractions of bound RNA were plotted against the protein concentrations. For calculating equilibrium dissociation constants $\left(K_{\mathrm{d}}\right)$, the plotted data were fit to the 1:1 binding equation using the Kaleida Graph software (Hulinks) as described before [23].

\subsection{Cell Culture}

NIH3T3 cells (RIKEN cell bank; RCB1862) were maintained in Dulbecco's modified Eagle's medium (DMEM) (Fujifilm Wako Pure Chemical) containing 10\% FBS.

\subsection{Western Blotting Assay}

Cells were lysed using the RIPA buffer, and cell lysates were subjected to SDS-PAGE. The proteins were detected using mouse anti-FLAG antibody (Sigma Aldrich; St. Louis, MO, USA, F3165) or mouse anti- $\beta$-actin antibody (Sigma Aldrich; AC-74) as primary antibodies, and Goat anti-mouse IgG antibody (GeneTex) as the Horseradish peroxidase (HRP)-labeled secondary antibody. The bands were visualized using an Amersham ECL Prime (GE Healthcare, Chicago, IL, USA).

\subsection{Luciferase Reporter Assay}

Here, $1.5 \times 10^{4}$ of NIH3T3 cells were seeded onto 96-well plates the day before transfection. Transfection was performed using Lipofectamine LTX (Thermo Fisher Scientific, Waltham, MA, USA). Transfection mixtures contained $100 \mathrm{ng}$ TTP-PUF expression vector and $1 \mathrm{ng}$ reporter vector. For control, pCMV-TTP(C147R)-GG-PUF (Addgene \#1000000051), in which the TTP gene but no PUF-encoding sequences were inserted, was used instead of TTP-PUF expression vectors. Then, $24 \mathrm{~h}$ after transfection, cells were lysed using Passive Lysis Buffer (Promega). Firefly luciferase and Renilla luciferase activities were measured using the Dual-Luciferase Reporter Assay System (Promega) with measurements taken on a GloMax-Multi Detection System (Promega). Firefly luciferase activity (Fluc) was normalized to Renilla luciferase activity (Rluc), and then relative luciferase activities (RLAs), compared to control 
samples, were calculated (Equation (1)). The degree of repression by a TTP-PUF relative to the control was indicated by the reciprocal of the relative luciferase activity (Equation (2)).

$$
\operatorname{RLA}_{(\text {TTP-PUF) }}=\left[\text { Fluc }_{(\text {TTP-PUF })} / \text { Rluc }_{(\text {TTP-PUF) }}\right] /\left[\text { Fluc }_{(\text {control })} / \text { Rluc }_{(\text {control })}\right]
$$

$$
\text { Fold repression }=1 / \text { RLA }
$$

\section{Results and Discussion}

\subsection{RNA-Binding Properties of Programmable RNA-Binding Proteins}

Four PUF proteins, PUF (A), (B), (C), and (D), targeting different RNA sequences, RNA (a), (b), (c) and (d), respectively, were designed by assembling PUF repeats corresponding to each RNA base in order (Figure 1d). EMSA revealed the high affinity and specificity of PUF proteins to target RNA sequences. In this assay, the binding of PUF proteins reduced the mobility of the target RNAs, allowing the evaluation of RNA-binding abilities of proteins. PUF (A)-(D) proteins were expressed in E. coli cells and purified via affinity chromatography using a Ni-NTA column. PUF (A), (B), (C), and (D) showed band-shifts for RNA (a), (b), (c), and (d), respectively (Figure 2, Figure S2a). The apparent dissociation constants between each PUF protein and corresponding RNA were $10.1 \mathrm{nM}$ (PUF (A)/RNA (a)), $5.3 \mathrm{nM}$ (PUF (B)/RNA (b)), $<1$ nM (PUF (C)/RNA (c)), and 9.1 nM (PUF (D)/RNA (d)) (Table 1, Figure S2b). Although the $K_{d}$ values were somewhat different between PUFs, they were within the reported range of engineered PUF proteins $\left(10^{-10}-10^{-8} \mathrm{M}\right)$ [23,31]. Importantly, all PUF (A)-(D) proteins showed high specificity to their corresponding RNA sequences (Figure S2a). RNA (a) showed clear shift bands only when incubated with PUF (A) but not with the other three PUFs. Similarly, RNA (b)-(d) showed clear shift bands only when incubated with their corresponding PUFs.

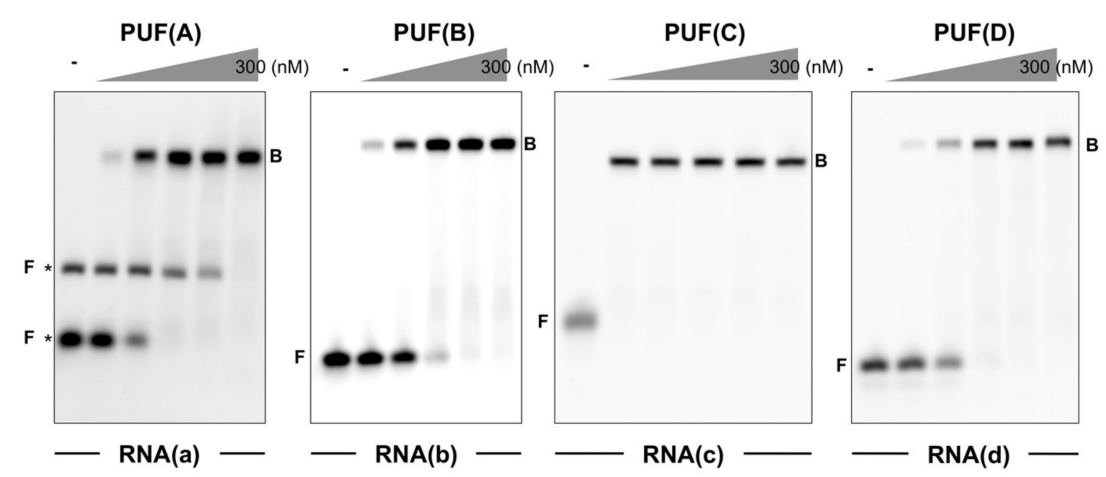

Figure 2. RNA binding of engineered PUF proteins to target RNAs. In the electrophoretic mobility shift assay (EMSA), RNA oligonucleotides, RNA (a)-(d), were incubated with corresponding PUF proteins, PUF (A)-(D), respectively, at the following concentrations (left to right): 0, 3.7, 11, 33, 100, 300 $\mathrm{nM}$, and the mixtures were electrophoresed in native PAGE. " $\mathrm{F}$ " and " $\mathrm{B}$ " indicate protein-free RNA and protein-bound RNA, respectively. RNA (a) showed two protein-free bands (shown by *).

Table 1. RNA binding of engineered PUF proteins to specific RNA sequences.

\begin{tabular}{ccccc}
\hline \multicolumn{5}{c}{$\boldsymbol{K}_{\mathbf{D}}(\mathbf{n M})^{\mathbf{1}}$} \\
\hline PUF (A) & RNA(a) & RNA(b) & RNA(c) & RNA(d) \\
\hline PUF (B) & $10.1 \pm 1.4$ & n.d. & n.d. & n.d. \\
PUF (C) & n.d. & $5.3 \pm 2.7$ & n.d. & n.d. \\
PUF (D) & n.d. & n.d. & $<1$ & n.d. \\
\hline \multicolumn{7}{c}{${ }^{1}$ mean \pm SD, n.d.; not detected. }
\end{tabular}




\subsection{Specific RNA Binding of TTP-PUF within Cells}

The RNA binding specificities of PUF (A)-(D) for their corresponding target RNAs were also confirmed within living cells using luciferase reporter assays (Figure 3, Figure S1a,b). PUF (A)-(D) fused with tristetraprolin (TTP), an mRNA decay factor, were designed as described before [23,31]. As a control, a TTP expression vector that expresses only TTP without fusing with PUFs was used to estimate the effect of PUF $[23,31]$. Previous reports showed that a TTP-PUF fusion protein, which can bind to the $3^{\prime}$ UTR of the luciferase reporter gene transcript, represses luciferase activity [23,31]. The degree of luciferase repression reflects the RNA binding of TTP-PUF fusion proteins. Here, we prepared reporter vectors that express both firefly luciferase and Renilla luciferase genes driven by a PGK and an SV40 promoter, respectively (Figure S1a,b). A four-times repeat sequence of the PUF target sequences, $4 \times(\mathrm{a}),(\mathrm{b}),(\mathrm{c})$ or (d), was inserted at the $3^{\prime}$ UTR of the firefly luciferase gene (Figure 3a). Each TTP-PUF expression or control vectors and a reporter vector were transfected into NIH3T3 cells. In this reporter system, we expected the firefly luciferase activity to be affected by TTP-PUFs, and Renilla luciferase activity to reflect the transfection efficiencies. Thus, the firefly luciferase activity, under the expression of each TTP-PUF, was normalized to the Renilla luciferase activity of the same sample. The normalized luciferase activity was compared to that of the control sample to evaluate the degree of repression. The expression of the TTP-PUF proteins was confirmed via Western blotting assay (Figure S1c). As shown in Figure 3b, TTP-PUF (A), (B), (C), and (D) showed a 4-6-fold repression in luciferase gene expression with corresponding RNA-binding sequences at the $3^{\prime}$ UTR. By contrast, they had little effect on luciferase gene expression without corresponding binding sites at the $3^{\prime}$ UTR. None of the TTP-PUFs repressed the luciferase activity in the no binding site (nbs) reporter. These results indicated that the PUF proteins specifically bound to the corresponding RNA sequences at the $3^{\prime}$ UTR of the luciferase gene even within cells.

(a)

TTP-PUF

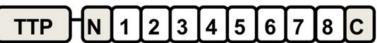

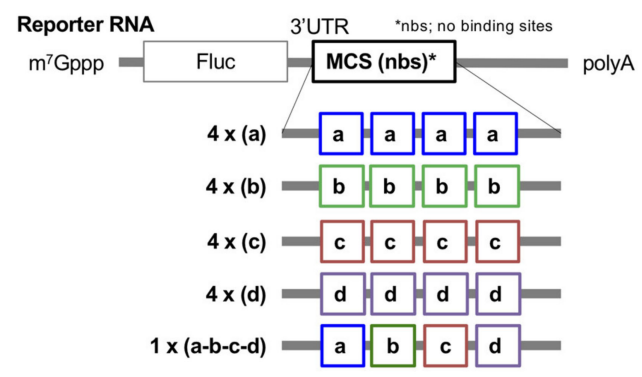

(b)

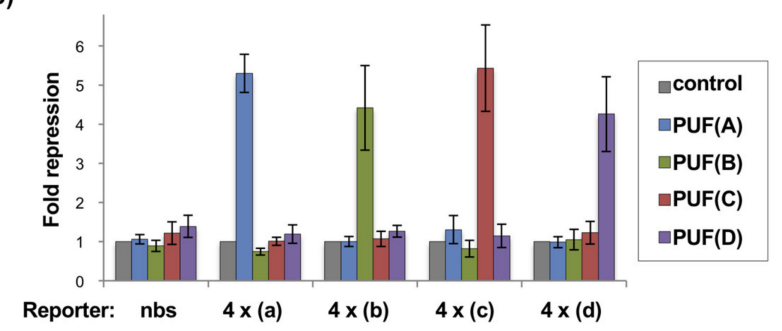

Figure 3. Specific binding of engineered PUF proteins to target RNAs in living cells. (a) Schematic representation of TTP-PUF proteins and reporter RNAs. TTP-PUF expression plasmids and luciferase reporter plasmids were co-transfected into NIH3T3 cells. The luciferase reporter genes were transcribed, and reporter RNAs with PUF-binding sequences at the $3^{\prime}$ untranslated region (UTR) region should have been produced in the cells. (b) Fold repression of luciferase reporter activities by TTP-PUF proteins. Data are presented as mean $\pm \mathrm{SD}$. 


\subsection{Effective Repression of Reporter Genes with Not-Repetitive Sequences by Multiple TTP-PUF Proteins}

It is unusual that four or more times sequences exist at the regulatory region of single transcripts. Instead of targeting repeat sequences at the $3^{\prime}$ UTR, the synergistic effect of multiple TTP-PUFs was shown through the targeting of multiple RNA sequences at the $3^{\prime}$ UTR. As a model of transcripts, a reporter vector with a $1 \times(a-b-c-d)$ sequence at the $3^{\prime}$ UTR of the firefly luciferase gene was designed, in which only one binding sequence for each PUF (A)-(D) existed at the $3^{\prime}$ UTR (Figure 3a). In contrast to the 4-6-fold repression of the luciferase genes with $4 \times$ binding sequences, each TTP-PUF (A), (C), or (D) showed only about a 1.5-fold repression (Figure 4, lanes 3,5,6), and TTP-PUF (B) alone did not repress luciferase activity (Figure 4, lane 4), maybe because of the low TTP-PUF (B) expression compared to other TTP-PUF proteins (Figure S1c).

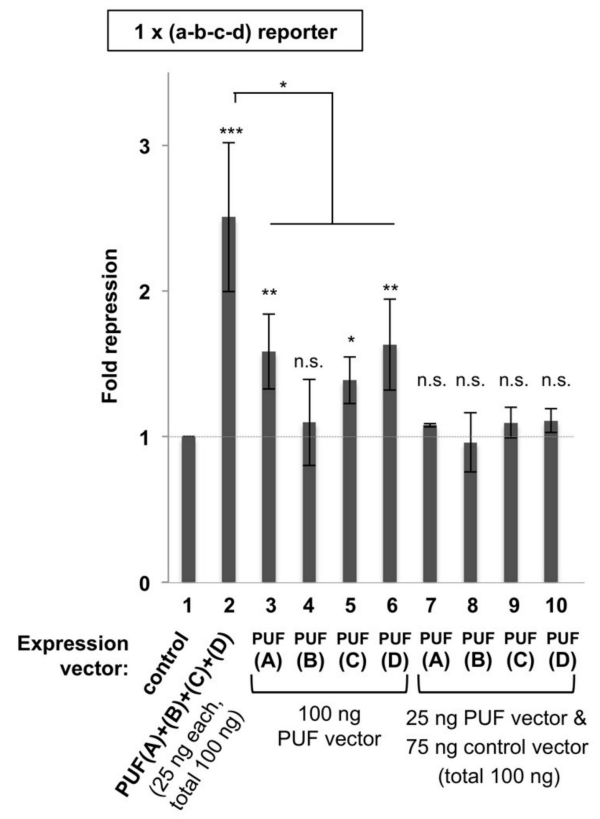

Figure 4. Combination of TTP-PUF (A)-(D) with $1 \times(a-b-c-d)$ reporter efficiently repressed the luciferase reporter activities at the $3^{\prime}$ UTR of the luciferase mRNA. NIH3T3 cells were co-transfected with $1 \times$ (a-b-c-d) reporter (1 ng) and $100 \mathrm{ng}$ (total) of the following expression vectors. Lane 1, control vector; lane 2, mixture of TTP-PUF (A), (B), (C), and (D) (25 ng each); lanes 3-6, $100 \mathrm{ng}$ of TTP-PUF (A) (lane 3), (B) (lane 4), (C) (lane 5), (D) (lane 6); lanes 7-10, $75 \mathrm{ng}$ of control vector and $25 \mathrm{ng}$ of TTP-PUF (A) (lane 7), (B) (lane 8), (C) (lane 9), (D) (lane 10). Data are presented as mean \pm SD. $(* * *, p<0.005 ; * *, p<0.01 ; *$, $p<0.05$; n.s. $=$ not significant)

When NIH3T3 cells were co-transfected with all of the expression vectors (TTP-PUF (A)-(D); $25 \mathrm{ng}$ each) and the $1 \times(\mathrm{a}-\mathrm{b}-\mathrm{c}-\mathrm{d})$ reporter vector, significant repression (2.5-fold) of luciferase activity was observed, even though the amount of each expression vector (25 ng each) was one-fourth of that when used alone (100 ng) (Figure 4, lane 2). The mixture of four TTP-PUF expression vectors had little effect on the reporter lacking a PUF-binding sequence (Figure S3). Furthermore, when the cells were transfected with $25 \mathrm{ng}$ of one of the four TTP-PUF vectors, the same amount used for the mixture, none of them showed significant repression of the $1 \times(a-b-c-d)$ reporter (Figure 4 , lanes 7-10). These results indicated that the luciferase activity of the $1 \times(a-b-c-d)$ reporter vector was repressed by the sequence-specific binding of TTP-PUF proteins to their binding sites at the 3' UTR. Additionally, the combination of four TTP-PUF proteins, targeting different RNA sequences, caused effective repression of luciferase activity compared to the use of a single TTP-PUF protein. Differences in repression ratios were observed between reporters with $4 \times$ binding sequences by the corresponding single TTP-PUF (4-6-fold) (Figure $3 b$ ) and the $1 \times(a-b-c-d)$ reporter by the mixture of TTP-PUF (A)-(D) (2.5-fold) (Figure 4). One plausible reason for the difference is avidity effects for the 
$4 \times$ proximity binding sites. The overall RNA structure of the PUF binding sequences can affect the binding of TTP-PUFs. Further optimization will increase the synergistic effects of multiple functional PUF proteins.

So far, synergistic gene activation or repression was shown by a combination of multiple artificial transcription regulators based on transcription activator-like effectors (TALEs) and CRISPR-dCas9 [32,33]. The results using a combination of multiple RNA binding proteins are in accordance with these reports targeting DNA.

\section{Conclusions}

In this study, four independent PUF proteins were designed and shown to recognize the target RNA sequence with high specificity both in vitro and in cellulo. By combining these four PUF proteins, we showed the use of multiple TTP-PUF proteins in the effective repression of target gene expression, even though the amount of each transfected expression vector was smaller than that of a single PUF protein. Although further verification is needed to understand the off-target effects, we expect that this strategy will reduce off-target effects because of a lower dose of expression vectors. Sequence-specific RNA-binding molecules, like PUFs, can exert desired functions at specific RNA regions when fused with various functional domains. As the importance of RNAs in life science becomes clear, there is an increasing demand for methods to control specific RNAs. Combination of multiple PUF proteins would be a promising strategy to control endogenous RNAs effectively. Furthermore, this concept might be applied to other RNA-targeting molecular tools, such as PPR proteins and CRISPR-Cas13 based systems.

Supplementary Materials: The following are available online at http://www.mdpi.com/2076-3417/10/19/6803/s1, Figure S1: Plasmids used for luciferase reporter assays and expression of TTP-PUFs, Figure S2: Representative results of EMSA, Figure S3: Effect of the mixture of TTP-PUFs on the nbs reporter.

Author Contributions: Conceptualization, M.I. and M.S.; investigation, M.S. and A.S.; writing-M.I. and S.F. All authors have read and agreed to the published version of the manuscript.

Funding: This research was funded by JSPS/KAKENHI (19H02850 to M.I.).

Acknowledgments: We kindly thank Huimin Zhao for plasmids to construct PUFs (Addgene \#1000000051).

Conflicts of Interest: The authors declare no conflict of interest.

\section{References}

1. Pei, Y.; Lu, M. Programmable RNA manipulation in living cells. Cell. Mol. Life Sci. 2019, 76, 4861-5867. [CrossRef] [PubMed]

2. Hall, T.M. De-coding and re-coding RNA recognition by PUF and PPR repeat proteins. Curr. Opin. Struct. Biol. 2016, 36, 116-121. [CrossRef] [PubMed]

3. Chen, Y.; Varani, G. Engineering RNA-binding proteins for biology. FEBS J. 2013, 280, 3734-3754. [CrossRef] [PubMed]

4. Terns, M.P. CRISPR-Based Technologies: Impact of RNA-Targeting Systems. Mol. Cell 2018, 72, 404-412. [CrossRef] [PubMed]

5. Filipovska, A.; Rackham, O. Designer RNA-binding proteins: New tools for manipulating the transcriptome. RNA Biol. 2011, 8, 978-983. [CrossRef]

6. Mackay, J.P.; Font, J.; Segal, D.J. The prospects for designer single-stranded RNA-binding proteins. Nat. Struct. Mol. Biol. 2011, 18, 256-261. [CrossRef]

7. Cao, J.; Arha, M.; Sudrik, C.; Schaffer, D.V.; Kane, R.S. Bidirectional regulation of mRNA translation in mammalian cells by using PUF domains. Angew. Chem. Int. Ed. 2014, 53, 4900-4904. [CrossRef]

8. Campbell, Z.T.; Valley, C.T.; Wickens, M. A protein-RNA specificity code enables targeted activation of an endogenous human transcript. Nat. Struct. Mol. Biol. 2014, 21, 732-738. [CrossRef] 
9. Dong, S.; Wang, Y.; Cassidy-Amstutz, C.; Lu, G.; Bigler, R.; Jezyk, M.R.; Li, C.; Hall, T.M.; Wang, Z. Specific and modular binding code for cytosine recognition in Pumilio/FBF (PUF) RNA-binding domains. J. Biol. Chem. 2011, 286, 26732-26742. [CrossRef]

10. Wang, Y.; Cheong, C.G.; Hall, T.M.; Wang, Z. Engineering splicing factors with designed specificities. Nat. Methods 2009, 6, 825-830. [CrossRef]

11. Du, M.; Jillette, N.; Zhu, J.J.; Li, S.; Cheng, A.W. CRISPR artificial splicing factors. Nat. Commun. 2020, 11, 2973. [CrossRef] [PubMed]

12. Cox, D.B.T.; Gootenberg, J.S.; Abudayyeh, O.O.; Franklin, B.; Kellner, M.J.; Joung, J.; Zhang, F. RNA editing with CRISPR-Cas13. Science 2017, 358, 1019-1027. [CrossRef] [PubMed]

13. Liu, X.M.; Zhou, J.; Mao, Y.; Ji, Q.; Qian, S.B. Programmable RNA N ${ }^{6}$-methyladenosine editing by CRISPR-Cas9 conjugates. Nat. Chem. Biol. 2019, 15, 865-871. [CrossRef] [PubMed]

14. Shinoda, K.; Suda, A.; Otonari, K.; Futaki, S.; Imanishi, M. Programmable RNA methylation and demethylation using PUF RNA binding proteins. Chem. Commun. 2020, 56, 1365-1368. [CrossRef]

15. Rau, K.; Roesner, L.; Rentmeister, A. Sequence-specific $\mathrm{m}^{6} \mathrm{~A}$ demethylation in RNA by FTO fused to RCas9. RNA 2019, 25, 1311-1323. [CrossRef]

16. Wilson, C.; Chen, P.J.; Miao, Z.; Liu, D.R. Programmable $\mathrm{m}^{6} \mathrm{~A}$ modification of cellular RNAs with a Cas13-directed methyltransferase. Nat. Biotechnol. 2020. [CrossRef]

17. Li, J.; Chen, Z.; Chen, F.; Xie, G.; Ling, Y.; Peng, Y.; Lin, Y.; Luo, N.; Chiang, C.M.; Wang, H. Targeted mRNA demethylation using an engineered dCas13b-ALKBH5 fusion protein. Nucleic Acids Res. 2020, 48, 5684-5694. [CrossRef]

18. Zhao, J.; Li, B.; Ma, J.; Jin, W.; Ma, X. Photoactivatable RNA N6 ${ }^{6}$-methyladenosine editing with CRISPR-Cas13. Small 2020, 16, e1907301. [CrossRef]

19. Wang, X.; Zamore, P.D.; Hall, T.M. Crystal structure of a Pumilio homology domain. Mol. Cell 2001, 7, 855-865. [CrossRef]

20. Wang, X.; McLachlan, J.; Zamore, P.D.; Hall, T.M. Modular recognition of RNA by a human pumilio-homology domain. Cell 2002, 110, 501-512. [CrossRef]

21. Filipovska, A.; Razif, M.F.; Nygard, K.K.; Rackham, O. A universal code for RNA recognition by PUF proteins. Nat. Chem. Biol. 2011, 7, 425-427. [CrossRef] [PubMed]

22. Cheong, C.G.; Hall, T.M. Engineering RNA sequence specificity of Pumilio repeats. Proc. Natl. Acad. Sci. USA 2006, 103, 13635-13639. [CrossRef] [PubMed]

23. Shinoda, K.; Tsuji, S.; Futaki, S.; Imanishi, M. Nested PUF proteins: Extending target RNA elements for gene regulation. Chembiochem 2018, 19, 171-176. [CrossRef] [PubMed]

24. Adamala, K.P.; Martin-Alarcon, D.A.; Boyden, E.S. Programmable RNA-binding protein composed of repeats of a single modular unit. Proc. Natl. Acad. Sci. USA 2016, 113, E2579-E2588. [CrossRef]

25. Zhao, Y.Y.; Mao, M.W.; Zhang, W.J.; Wang, J.; Li, H.T.; Yang, Y.; Wang, Z.; Wu, J.W. Expanding RNA binding specificity and affinity of engineered PUF domains. Nucleic Acids Res. 2018, 46, 4771-4782. [CrossRef]

26. Criscuolo, S.; Gatti Iou, M.; Merolla, A.; Maragliano, L.; Cesca, F.; Benfenati, F. Engineering REST-specific synthetic PUF proteins to control neuronal gene expression: A combined experimental and computational study. ACS Synth. Biol. 2020, 9, 2039-2054. [CrossRef]

27. Ozawa, T.; Natori, Y.; Sato, M.; Umezawa, Y. Imaging dynamics of endogenous mitochondrial RNA in single living cells. Nat. Methods 2007, 4, 413-419. [CrossRef]

28. Yoshimura, H.; Inaguma, A.; Yamada, T.; Ozawa, T. Fluorescent probes for imaging endogenous beta-actin mRNA in living cells using fluorescent protein-tagged pumilio. ACS Chem. Biol. 2012, 7, 999-1005. [CrossRef]

29. Yamada, T.; Yoshimura, H.; Inaguma, A.; Ozawa, T. Visualization of nonengineered single mRNAs in living cells using genetically encoded fluorescent probes. Anal. Chem. 2011, 83, 5708-5714. [CrossRef]

30. Ozawa, T.; Umezawa, Y. Genetically-encoded fluorescent probes for imaging endogenous mRNA in living cells. Methods Mol. Biol. 2011, 714, 175-188.

31. Abil, Z.; Denard, C.A.; Zhao, H. Modular assembly of designer PUF proteins for specific post-transcriptional regulation of endogenous RNA. J. Biol. Eng. 2014, 8, 7. [CrossRef] [PubMed] 
32. Nomura, W.; Matsumoto, D.; Sugii, T.; Kobayakawa, T.; Tamamura, H. Efficient and orthogonal transcription regulation by chemically inducible artificial transcription factors. Biochemistry 2018, 57, 6452-6459. [CrossRef] [PubMed]

33. Perez-Pinera, P.; Ousterout, D.G.; Brunger, J.M.; Farin, A.M.; Glass, K.A.; Guilak, F.; Crawford, G.E.; Hartemink, A.J.; Gersbach, C.A. Synergistic and tunable human gene activation by combinations of synthetic transcription factors. Nat. Methods 2013, 10, 239-242. [CrossRef] [PubMed] 